\title{
Detection of Microsatellite Instability via Circulating Tumor DNA and Response to Immunotherapy in Metastatic Castration- Resistant Prostate Cancer: A Case Series
}

\author{
Deepak Ravindranathan $^{a} \quad$ Greta Anne Russler $^{\mathrm{a}} \quad$ Lauren Yantorni $^{\mathrm{a}}$ \\ Leylah M. Drusbosky ${ }^{b}$ Mehmet Asim Bilen ${ }^{a}$ \\ aDepartment of Hematology and Medical Oncology, Winship Cancer Institute of Emory \\ University, Atlanta, GA, USA; 'buardant Health, Inc, Redwood City, CA, USA
}

\section{Keywords}

Prostate cancer $\cdot$ Immunotherapy $\cdot$ Liquid biopsy $\cdot$ Microsatellite instability

\begin{abstract}
Pembrolizumab has been approved by the US Food and Drug Administration for the treatment of metastatic or unresectable solid tumors that are microsatellite instability-high (MSI$\mathrm{H}$ ) or mismatch repair deficient. Blood-based circulating tumor DNA (ctDNA) assays have been validated to identify tumors with MSI-H status without the need for tissue biopsy. We report 2 patients with metastatic castration-resistant prostate cancer (mCRPC) who had prior treatment with multiple lines of therapy and underwent ctDNA testing, which detected MSI-H status. Both patients were treated with pembrolizumab, resulting in an excellent clinical response measured with liquid biopsies before and after initiation of therapy, which demonstrated a significant reduction in somatic-variant allele frequency in addition to a decrease in prostate serum antigen levels.

\section{Introduction}

Initial treatment for metastatic prostate cancer involves androgen deprivation therapy; however, disease progression to a castration-resistant state is inevitable. Pembrolizumab, an antibody targeting the programmed cell death protein, PD-1, was approved by the US Food and Drug Administration (FDA) for the treatment of solid tumors with microsatellite instability (MSI) or mismatch repair deficiency (dMMR), regardless of primary origin [1]. 
Ravindranathan et al.: ctDNA Detection of MSI-H Prostate Cancer and Response to Pembrolizumab

MSI-H or dMMR has been found in $2-12 \%$ of metastatic prostate cancer cases [2]. Typically, molecular testing is performed on tissue biopsy samples, which undergo immunostaining or genomic analysis by PCR or DNA sequencing techniques. However, routine biopsy of metastatic castration-resistant prostate cancer (mCRPC) metastases may not be feasible due to cost, comorbidity, and low yield due to the bone-predominant metastatic spread of prostate cancer, occurring in 30\% of patients within 2 years of castration resistance and in over $90 \%$ of patients over the course of the disease [3]. Alternatively, commercial circulating tumor DNA (ctDNA) detection assays, such as Guardant360 (Guardant Health, Inc., Redwood City, CA, USA), perform deep sequencing of at least 70 clinically relevant genes [4]. This approach can also be utilized to test for MSI-H status without the need for tissue biopsy via a simple blood draw. In fact, the Guardant360 liquid biopsy assay has shown high concordance with matched tissue samples in nearly 1,000 patients [5]. Tissue biopsy is reflective of mutations and information of a single site at a certain time point of the cancer, while liquid biopsy can give real-time information as it allows for analysis of clonal and subclonal variants [6].

Here, we present 2 consecutive cases of ctDNA-informed MSI-H mCRPC patients cared for at our institution and treated with pembrolizumab. Both cases showed an excellent response, which was demonstrated by liquid biopsies that were obtained prior to the start of immunotherapy and after 5 cycles of treatment, showing reduction and clearance of somatic mutations.

\section{Case Reports}

\section{Case 1}

We present a 51-year-old African American male with metastatic prostate adenocarcinoma (Gleason score of $5+5=10$ ) diagnosed in December 2016. The patient initially received bicalutamide and then started on leuprolide injections every 3 months, in combination with docetaxel. The prostate serum antigen (PSA) level at the time of diagnosis was $353 \mathrm{ng} / \mathrm{mL}$ (Fig. 1A, a). The patient underwent 3 cycles of docetaxel, and further treatment was stopped due to a significant infusion reaction. His PSA continued to rise, and he was then started on abiraterone and prednisone (Fig. 1A, d). Within 4 weeks of the initiation of therapy, his PSA quadrupled (Fig. 1A, e). At that time, abiraterone was stopped and the patient was started on enzalutamide, and his PSA decreased. However, the patient's PSA increased over the next 6 months, causing concern for progression of disease (Fig. 1A, f). This prompted MRI of the patient's abdomen and pelvis (Fig. 2A, B), which showed a new 11.5-cm abnormal soft tissue mass encasing the left renal pelvis and proximal left ureter, and a $2.5-\mathrm{cm}$ mass at the interface between the prostate and left posterior aspect of the urinary bladder.

Biopsy of the 11.5-cm abnormal soft tissue mass revealed transformed, poorly differentiated prostatic adenocarcinoma. The patient then underwent 6 cycles of carboplatin and etoposide, resulting in a partial response. However, PSA started to increase again (Fig. 1A, g). Given the history of significant reaction to taxanes, options for the next line of therapy were limited. This prompted us to send peripheral blood for Guardant360, which identified MSI-H status via digital NGS testing (Fig. 1B). The patient was started on pembrolizumab $(200 \mathrm{mg}$ every 3 weeks intravenously). His PSA was $39.90 \mathrm{ng} / \mathrm{dL}$ prior to the first cycle of pembrolizumab (Fig. 1A, h) and it decreased to $4.9 \mathrm{ng} / \mathrm{dL}$ after completing the first cycle. He continued on leuprolide injections every 3 months and has thus far undergone 12 cycles of pembrolizumab without experiencing any adverse events. At the time of this publication, his PSA is undetectable $(<0.01 \mathrm{ng} / \mathrm{dL})$. All of the PSA levels since diagnosis are shown in Figure 1. Recent MRI of the patient's abdomen and pelvis showed no measurable residual mass (Fig. 2C, D).

\section{Karger'}


Fig. 1. A Trend in PSA levels after diagnosis for case 1. a: initiation of leuprolide and C1D1 docetaxel; b: C3D1 of docetaxel (further cycles were stopped); c: initiation of abiraterone; d: discontinuation of abiraterone and initiation of enzalutamide; e: MRI of the abdomen and pelvis revealed a new left renal and prostatic mass; f: C1D1 of carboplatin and etoposide; g: C6D1 of carboplatin and etoposide; h: C1D1 of pembrolizumab. B Liquid biopsy results of case 1 demonstrating MSI-H status in (A), and clearance of somatic mutations and MSI-H status in (B). C Trend in PSA levels after diagnosis for case 2. a: initiation of leuprolide and EBRT; b: diagnosis of recurrence; c: initiation of enzalutamide; d: discontinuation of enzalutamide and initiation of abiraterone; e: administration of sipuleucel-T; $\mathrm{f}$ : C1D1 of docetaxel; g: C1 of Rad-223; h: C1D1 of pembrolizumab. D Liquid biopsy results of case 2 demonstrating MSI-H status in (A), and significant reduction of somatic variants and MSI-H status in (B). MSI-H, microsatellite instabilityhigh; ND, not detected; PSA, prostate serum antigen.

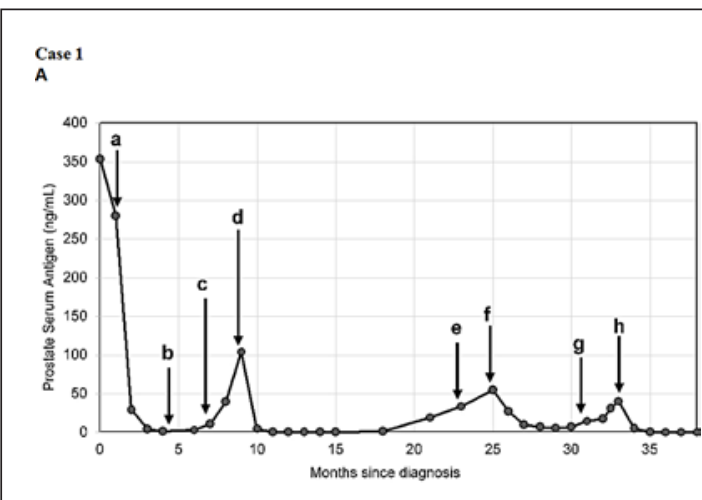

B

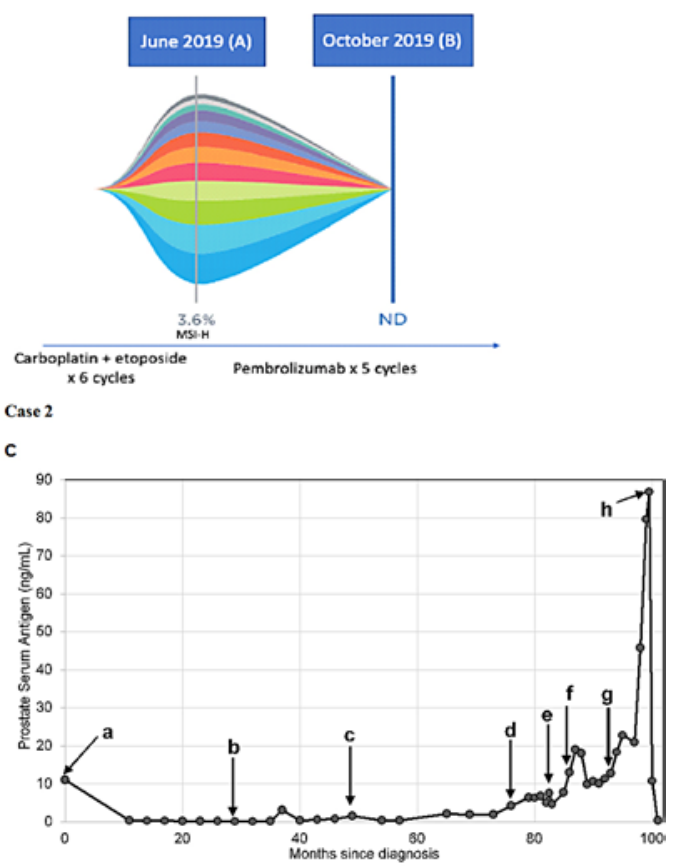

D

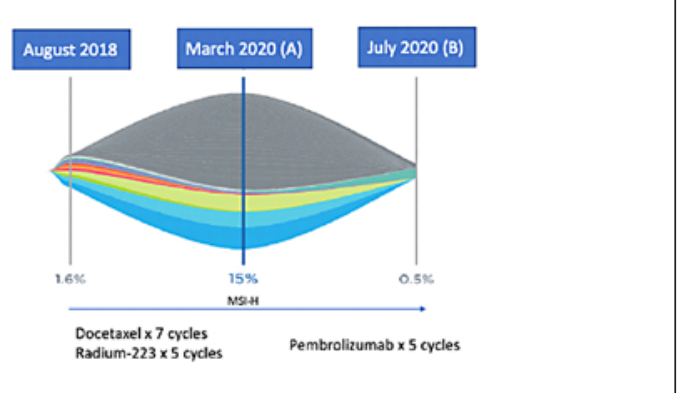

Repeat liquid biopsy analysis after 5 cycles of pembrolizumab showed clearance of somatic alterations and MSI-H status (Fig. 1B).

Case 2

Next we present an 81-year-old Caucasian male with metastatic prostate adenocarcinoma diagnosed in 2011 (Gleason score of $4+4=8$ ) who started on leuprolide injections and received proton therapy to the prostate. The PSA level at diagnosis was $11 \mathrm{ng} / \mathrm{mL}$ (Fig. 1C, 

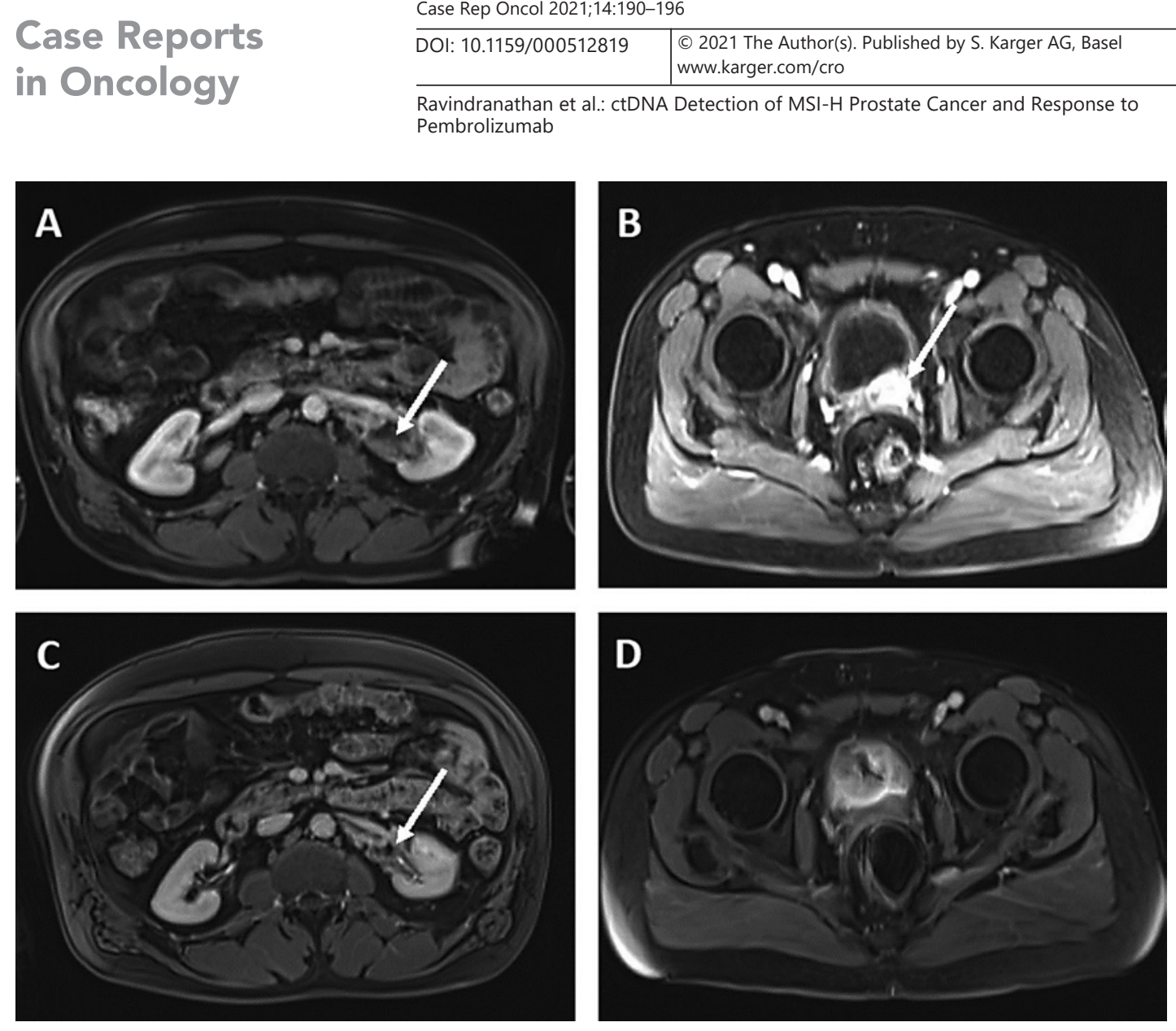

Fig. 2. MRI of the patient's abdomen and pelvis in case 1, showing a response of the left renal mass and prostatic mass to immunotherapy. A, B Soft tissue mass by left ureter (A) and prostatic mass (B) (shown by white arrows) before treatment with pembrolizumab. C, D Reduction in soft tissue mass (C) and no residual left prostatic mass (D) seen after 10 cycles of pembrolizumab.

a). About 2.5 years later, a bone scan confirmed recurrence with bone lesions at the left iliac spine and right posterior sixth rib (Fig. 1C, b). PSA was $1.46 \mathrm{ng} / \mathrm{mL}$ and he was started on enzalutamide (Fig. 1C, c). After 2 years, PSA increased to $4.18 \mathrm{ng} / \mathrm{mL}$ and the patient was started on abiraterone with prednisone (Fig. 1C, d). Six months later, the PSA continued to rise to $7.52 \mathrm{ng} / \mathrm{mL}$ and the patient received sipuleucel-T, but he experienced an allergic reaction and further treatment was halted per the patient's request (Fig. 1C, e). Later, docetaxel was initiated (Fig. 1C, f) and the patient underwent 7 cycles. He initially experienced disease control, and later, PSA increased to $18.31 \mathrm{ng} / \mathrm{mL}$ (Fig. 1C, g). Subsequently, he was treated with 5 cycles of radium-223; however, the PSA level continued to increase to $86.83 \mathrm{ng} / \mathrm{mL}$ (Fig. 1C, h). At that time, given metastatic castration-resistant disease and with limited options for further control of disease, Guardant360 was conducted and detected MSI-H status (Fig. 1D). The patient was promptly started on pembrolizumab (200 mg every 3 weeks intravenously). At the time of this publication, the patient has received 5 cycles of immunotherapy with an excellent response, and the PSA level last measured was $0.11 \mathrm{ng} / \mathrm{mL}$. Repeat liquid biopsy analysis after 5 cycles of pembrolizumab showed a significant reduction in somatic variant allele frequency, from 15 to $0.5 \%$, and clearance of MSI-H status from the sample (Fig. 1D). 


\section{Discussion}

We presented 2 cases of MCRPC with MSI-H status detected via ctDNA and treated with pembrolizumab, with excellent responses. The MMR system of DNA is a highly conserved mechanism that helps to identify and repair mismatched nucleotides resulting from recombination or damage from external stresses. This system consists of DNA MMR enzymes based on four main genes: mutL homologue 1 (MLH1), postmeiotic segregation increased 2 (PMS2), mutS homologue (MSH2), and mutS 6 (MSH6). These four proteins are typically detected through immunohistochemical staining of tissue samples. Inactivation of MMR genes or MMR protein dysfunction may induce MSI. MSI-H status is observed when there are more than two altered mononucleotide repeats.

The KEYNOTE-016, 164, 012, 028, and 158 trials showed good responses to pembrolizumab in tumors with dMMR or MSI-H status. Given the results from the trials, pembrolizumab was approved by the FDA for the treatment of unresectable or metastatic dMMR/ MSI-H tumors regardless of the primary tumor site, and this was one of the first pan-tumor biomarkers defined regardless of the primary tumor site in oncology [7]. Le et al. [8] reported that dMMR tumors have higher infiltration of CD8+ tumor-infiltrating lymphocytes, which harnesses immunotherapy to result in a robust response.

Identification of MSI-H prostate cancers and response rates to PD- 1 inhibitors is sparse in the literature. Tucker et al. [9] conducted a retrospective study and identified 48 men who received more than 1 cycle of pembrolizumab for metastatic castration-resistant disease. Of these, 45 men had more than 3 prior lines of therapy for metastatic castrationresistant disease and 4 patients had a greater than 90\% PSA decline with pembrolizumab, which was also observed in our 2 patients. Of those 4 men, one had MSI-H status [9]. Manogue et al. [10] reported one case of complete remission with pembrolizumab therapy in an mCRPC patient with an MSH2 alteration identified via tissue sampling. The potential utility of ctDNA to assess mutational burden was also demonstrated in that case. In patients with serial tumor assessments for MSI, 3 out of 5 patients acquired MSI in a second or subsequent sample [10]. Barata et al. [11] recently reported 14 patients from a multi-institutional review of patients with MSI-H status detected by ctDNA, out of whom 9 patients received pembrolizumab and 4 patients achieved a 50\% reduction in PSA level after a median of 4 weeks after treatment and 3 patients achieved a greater than $99 \%$ reduction. All patients reported in the study who were treated with pembrolizumab had ctDNA testing while having $\mathrm{mCRPC}$ and 0.6 months prior to starting pembrolizumab [11]. In our case series, we report ctDNA testing that was done before starting and during immunotherapy, which showed a complete clearance of somatic variants in case 1 (Fig. 1B) and a significant reduction in case 2 (Fig. 1D) in addition to a reduction of greater than $90 \%$ in PSA level in both cases within the same time frame.

Treatment with immunotherapy for both patients in this report was based on the ctDNA assay results. Cases reported in the literature are usually based on mutational analyses from tumor tissue. The limited options of subsequent lines of therapy for these patients prompted the use of liquid biopsy to interrogate genomic targets for drugs, including MSI. Tissue sampling is not feasible in many metastatic prostate cancer patients, as most of the time, patients may only present with bone metastases. Liquid biopsy is easier to obtain and can be readily repeated when patients demonstrate clinical signs of disease progression. In these 2 cases, we showed that liquid biopsy can also demonstrate response to treatment. These cases support the utilization of a widely available, commercial liquid biopsy assay in routine clinical practice.

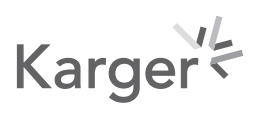




\section{Case Reports in Oncology}

\begin{tabular}{l|l}
\hline Case Rep Oncol 2021;14:190-196 \\
\hline DOI: 10.1159/000512819 & $\begin{array}{l}\text { ○ 2021 The Author(s). Published by S. Karger AG, Basel } \\
\text { www.karger.com/cro }\end{array}$ \\
\hline
\end{tabular}

Ravindranathan et al.: ctDNA Detection of MSI-H Prostate Cancer and Response to Pembrolizumab

\section{Conclusions}

Pembrolizumab has been approved to treat MSI-H tumors regardless of tumor type. Here, we have reported 2 cases of mCRPC with MSI-H status, detected via ctDNA, leading to treatment with pembrolizumab and resulting in an excellent clinical response as demonstrated by liquid biopsies collected before and after initiation of immunotherapy.

\section{Acknowledgements}

We like to thank Anthea Hammond for editing the manuscript.

\section{Statement of Ethics}

The subjects of this case report gave informed consent to publish their case including the publication of images. No institutional review board or other approvals were required.

\section{Conflict of Interest Statement}

M.A. Bilen has acted as a paid consultant for and/or as a member of the advisory boards of Exelixis, Bayer, BMS, Eisai, Pfizer, AstraZeneca, Janssen, Genomic Health, Nektar, and Sanofi and has received grants to his institution from Xencor, Bayer, Bristol-Myers Squibb, Genentech/Roche, Seattle Genetics, Incyte, Nektar, AstraZeneca, Tricon Pharmaceuticals, Peleton Therapeutics, and Pfizer for work performed as outside of the current study.

\section{Funding Sources}

No outside funding was solicited or obtained to produce this case report.

\section{Author Contributions}

M.A.B., L.Y., and G.A.R. cared for the patients. D.R. wrote the manuscript, which was edited by L.M.D. and M.A.B. All authors read and approved the final manuscript.

\section{References}

1 Boyiadzis MM, Kirkwood JM, Marshall JL, Pritchard CC, Azad NS, Gulley JL. Significance and implications of FDA approval of pembrolizumab for biomarker-defined disease. J Immunother Cancer. 2018;6(1):35.

2 Abida W, Cheng ML, Armenia J, Middha S, Autio KA, Vargas HA, et al. Analysis of the prevalence of microsatellite instability in prostate cancer and response to immune checkpoint blockade. JAMA Oncol. 2019;5(4): 471-8.

3 Den RB, George D, Pieczonka C, McNamara M. Ra-223 treatment for bone metastases in castrate-resistant prostate cancer: practical management issues for patient selection. Am J Clin Oncol. 2019;42(4):399-406.

4 Zill OA, Banks KC, Fairclough SR, Mortimer SA, Vowles JV, Mokhtari R, et al. The landscape of actionable genomic alterations in cell-free circulating tumor DNA from 21,807 advanced cancer patients. Clin Cancer Res. 2018;24(15):3528-38.

5 Willis J, Lefterova MI, Artyomenko A, Kasi PM, Nakamura Y, Mody K, et al. Validation of microsatellite instability detection using a comprehensive plasma-based genotyping panel. Clin Cancer Res. 2019;25(23):703545 . 
6 Moreno JG, Gomella LG. Evolution of the liquid biopsy in metastatic prostate cancer. Urology. 2019;132:1-9.

7 Zhao P, Li L, Jiang X, Li Q. Mismatch repair deficiency/microsatellite instability-high as a predictor for antiPD-1/PD-L1 immunotherapy efficacy. J Hematol Oncol. 2019;12(1):54.

8 Le DT, Uram JN, Wang H, Bartlett BR, Kemberling H, Eyring AD, et al. PD-1 blockade in tumors with mismatchrepair deficiency. N Engl J Med. 2015;372(26):2509-20.

9 Tucker MD, Zhu J, Marin D, Gupta RT, Gupta S, Berry WR, et al. Pembrolizumab in men with heavily treated metastatic castrate-resistant prostate cancer. Cancer Med. 2019;8(10):4644-55.

10 Manogue C, Cotogno P, Ledet E, Lewis B, Wyatt AW, Sartor O. Biomarkers for programmed death-1 inhibition in prostate cancer. Oncologist. 2019;24(4):444-8.

11 Barata P, Agarwal N, Nussenzveig R, Gerendash B, Jaeger E, Hatton W, et al. Clinical activity of pembrolizumab in metastatic prostate cancer with microsatellite instability (MSI-H) detected by circulating tumor DNA. J Immunother Cancer. 2020;8(2):e0001065. 